\title{
SEM Observation of Microcrystals Developed over Black Secretion on the Cultured Tissue of the Pearl Oyster Pinctada fucata
}

\author{
Tetsuro Samata, ${ }^{* 1}$ Hiroaki Somiya, ${ }^{* 2}$ Chie Horita, ${ }^{* 3}$ and Shusaku Akera ${ }^{* 3}$ \\ ${ }^{* 1}$ Department of Aquatic Biology, Research Institute of Biosciences, Azabu University, Fuchinobe, Sagamihara, \\ Kanagawa 229, Japan \\ ${ }^{* 2}$ Department of Animal Environment and Production, Faculty of Veterinary Medicine, Azabu University, Fuchinobe, \\ Sagamihara, Kanagawa 229, Japan \\ ${ }^{* 3}$ Tasaki Institute for Marine Biological Research, Tasaki Shinjyu Co., Ltd., Hiwasa, Kaifu, Tokushima 779-23, Japan \\ (Received September 24, 1993)
} mantle

Key words: pearl oyster, black secretion, SEM/EDS analysis, crystal formation, organ culture

Long-term culture of the mantle-epithelium of the pearl oyster is now possible following recent improvements in the culture medium, ${ }^{1,2)}$ and several interesting facts have been reported on the initial phase of shell formation. ${ }^{3-5)}$ This paper describes observations using scanning electron microscopy (SEM) and analysis by an energy dispersive X-ray microanalyser (EDS) on the microcrystals developed over the surface of the black secretion on the cultured tissue of Japanese pearl oysters.

The tissue piece was removed from the mantle epithelium of the pearl oyster Pinctada fucata, collected at Nagasaki, Japan. It was then cultured in an incubator, gassed with $10 \% \mathrm{CO}_{2}$ at $23^{\circ} \mathrm{C}$, at a $\mathrm{pH}$ of 7.2 to 7.6. The culture medium $\mathrm{Pf} 35$, developed for the cell culture of pearl oyster ${ }^{5)}$ was used. The cultured pieces were sampled and observed by SEM 15 to 30 days after the beginning of the culture. They were first washed repeatedly with distilled water, then the black secretion was separated carefully from the tissue using forceps. The black secretion was dried thoroughly and analyzed by an EDS (JEOL-JED2001), while part of the dried samples were coated with gold by ion-sputtering (JEOL-JFC1100E), and observed by an SEM (JEOL-JSM $5400 \mathrm{LV})$.

Microcrystals of various shapes and sizes, containing large amounts of $\mathrm{Ca}$ and $\mathrm{S}$, were detected in all the specimens examined. The specimens were classified into three groups according to the shape, size and composition: 1) flat petal-shaped crystals, $1-2 \mu \mathrm{m}$ in width and $5-20 \mu \mathrm{m}$ in length (Fig. 1A), 2) round to quadrilateral crystals with round edges, about $1 \mu \mathrm{m}$ in diameter (Fig. 1B), and 3) large round crystals resembling an assembly of smaller round crystals, larger than $50 \mu \mathrm{m}$ in diameter (Fig. 1C). The proportion of $\mathrm{Ca}$ to $\mathrm{S}$ showed a regular pattern of increase from the crystal of type 1 to type 3 (Fig. 2A to 2C). The type 3 crystals were particularly rich in $\mathrm{Ca}$ (Fig. 2C).

It is of great interest that the black secretion may have the capability to induce crystal growth, since crystals were distributed only over the area of it. Machii and Wada, ${ }^{5}$ who found the crystals on the black secretion, regarded them as a prismatic layer of calcite based on their properties such as shape, chemical composition and physical nature (birefringency). The present grouping of crystals differs distinctly from that reported in the previous paper ${ }^{5}$ not only by its morphological diversity but also by its size and physical nature (no birefringency). Moreover, sulfur was detected in all crystals examined, and the amount and ratio to calcium was dependent on the type of crystal. Based on the experimental observations in the initial phase of mineralization of the nacreous layer, Wada ${ }^{6,7)}$ reported that the nucleation and growth of crystals occurred only in a specific type of organic granule, which may be composed of glycoprotein with sulfate groups. The crystals found may be identical in nature to the substrates combined with the organic granules and the crystals described by Wada. ${ }^{6,7)}$ Therefore, further experiments based on a precise tracing of the process of the crystal nucleation and crystal growth on the black secretion will yield important information on the initial mechanisms of how the shell of the pearl oyster forms.

We would like to thank Mr. M. Maltby of the University of Cardiff, Great Britatin, for his advice on this project.

\section{References}

1) A. Machii: Bull. natn. Pearl Res. Lab., 8, $2111-2117$ (1974).

2) S. Kawai, A. Machii, and S. Kitamura: Ann. Rep. Osaka City Inst. Public Health Environ. Sci, 44, 64-72 (1981).

3) I. Yana and A. Machii: Bull. japan Soc. scient. Fish., 41, 1083 (1975).

4) A. Machii: Tissue Cult. Res. Commun., 4, 18-20 (1985).

5) A. Machii and K. Wada: in "Invertebrate Cell System Applications" (ed. by J. Mitsuhashi), CRC Press, Boca Raton, Florida, 1989, pp. 226-233.

6) K. Wada; Bull. natn. Pearl Res, Lab., 703-828 (1961).

7) K. Wada: Japan J. Crystal Growth, 12, 57-70 (1985). 


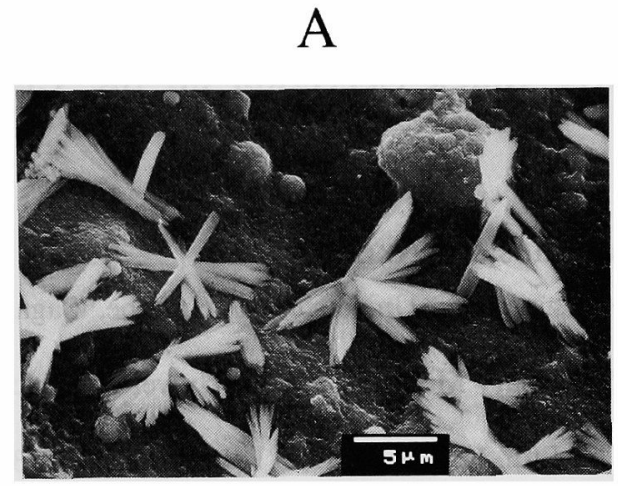

B

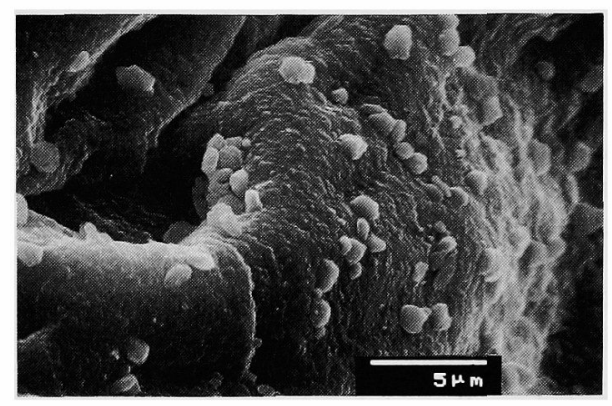

$\mathrm{C}$

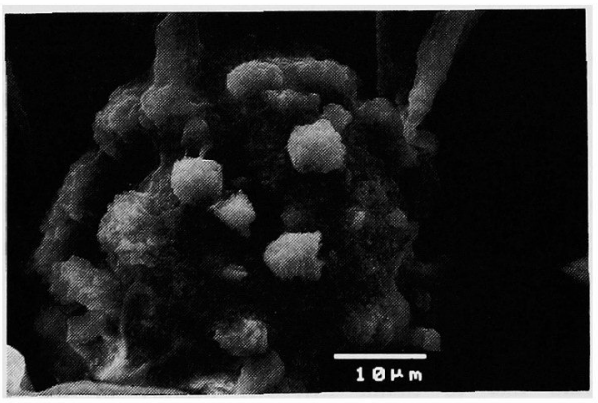

Fig. 1. Microcrystals developed on the surface of black secretion on the cultured tissue of the pearl oyster epithelium.

A, petal-shaped crystals; B, round to quadrillateral crystals with round edges; $\mathrm{C}$, large round crystals.

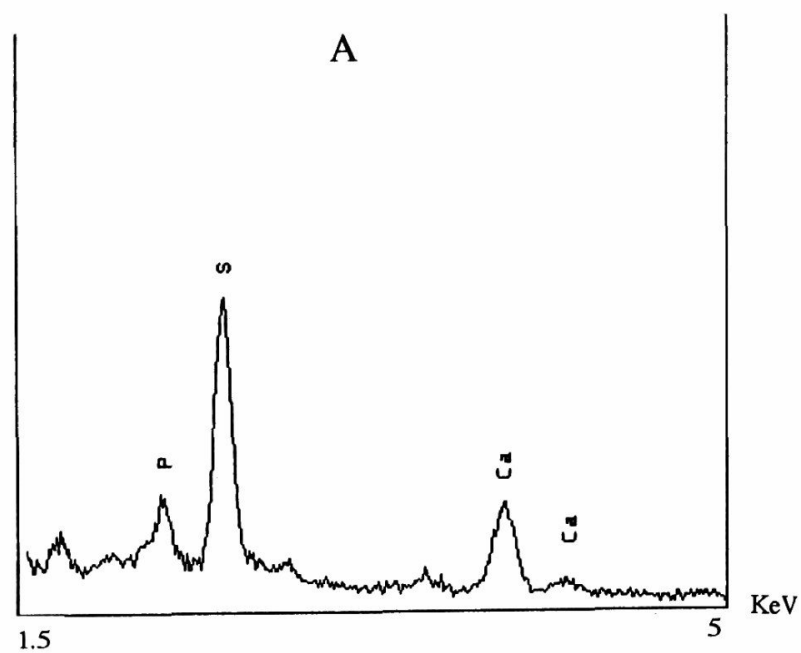

B
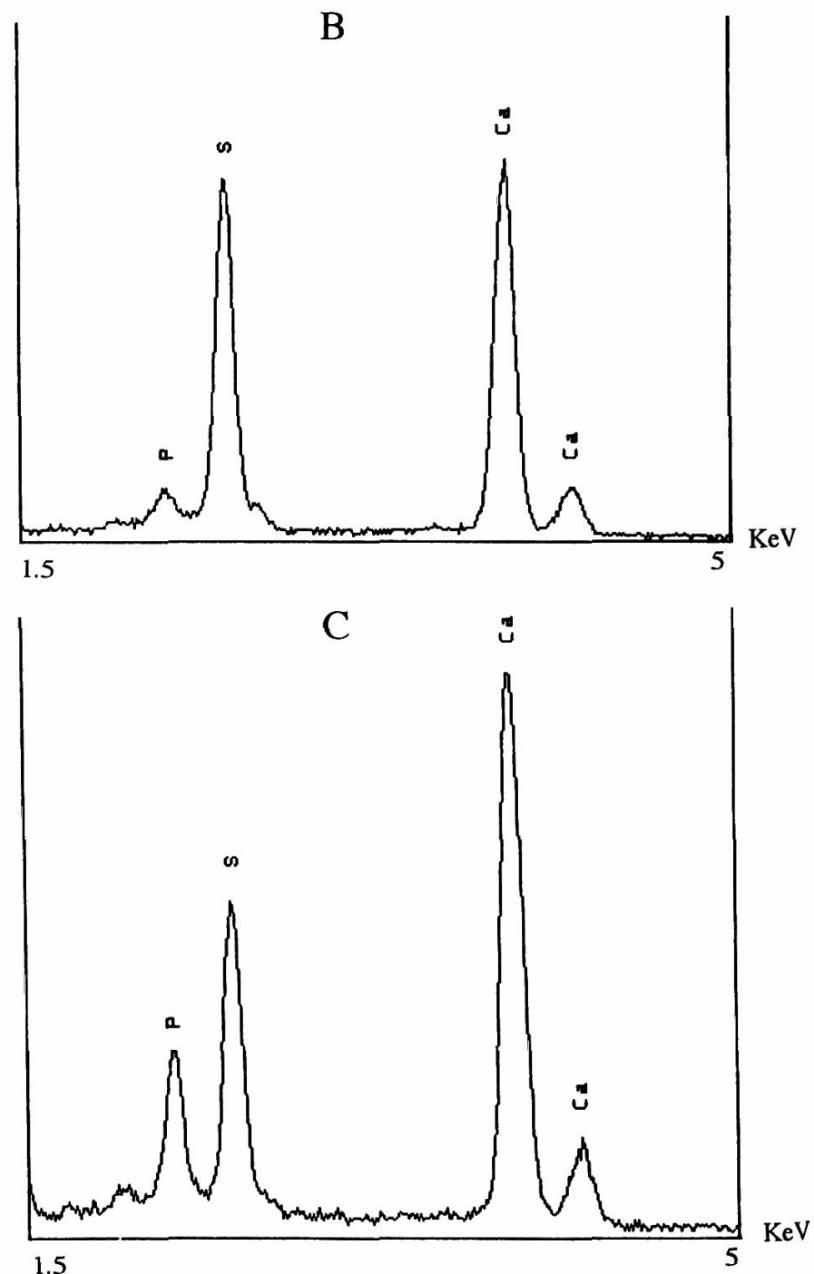

Fig. 2. X-ray spectrum of the microcrystals, which show a gradual change in the amount of $\mathrm{S}$ and $\mathrm{Ca}$.

$\mathrm{A}, \mathrm{B}$, and $\mathrm{C}$ correspond to the type of microcrystal in Fig. 1. 